Article

\title{
Effects of New Dietary Fiber from Japanese Apricot (Prunus mume Sieb. et Zucc.) on Gut Function and Intestinal Microflora in Adult Mice
}

\section{Motoi Tamura $^{1, *}$, Yuriko Ohnishi ${ }^{2}$, Tatsuya Kotani ${ }^{2}$ and Nobuki Gato ${ }^{2}$}

1 National Food Research Institute, The National Agriculture and Food Research Organization, Tsukuba, Ibaraki, Japan

2 Food Science Research Laboratory, Nakano BC Co. Ltd., Kainan, Wakayama, Japan; E-Mails: oonishi@nakano-group.co.jp (Y.O.); kotani@nakano-group.co.jp (T.K.); gato@nakano-group.co.jp (N.G.)

* Author to whom correspondence should be addressed; E-Mail: motoita@affrc.go.jp; Tel.: +81-298-38-8089; Fax: +81-298-38-7996.

Received: 18 February 2011; in revised form: 11 March 2011 / Accepted: 15 March 2011 / Published: 25 March 2011

\begin{abstract}
Much attention has been focused recently on functional foods. Ume, the Japanese name for the apricot of Prunus mume Sieb. et Zucc., is an example of a Japanese traditional functional food. There are, however, few reports on the effects of fiber from this fruit on bowel function. With this objective, we prepared ume fiber to test the hypothesis that it can change gut function and intestinal flora in mice. Mice were fed an ume fiber (UF) or cellulose (CF) diet (control) for 40 days. The fecal weight, fecal lipids, plasma lipids and cecal composition of the microflora were analyzed. The amount of feces was significantly greater in the UF group than in the CF group $(\mathrm{p}<0.01)$. The fecal lipids content $(\% \mathrm{DW})$ of the feces sampled on the final day of the experiment were significantly greater in the UF group than in the CF group $(\mathrm{p}<0.01)$. Plasma non-esterified fatty acids (NEFA) concentrations tended to be lower in the UF compared to the CF group $(\mathrm{p}=0.058)$. Occupation ratios of Bacteroides and Clostridium cluster IV were significantly greater in the cecal flora of the UF group. Our results suggest that ume fiber possesses the fecal lipid excretion effects and feces bulking effects.
\end{abstract}

Keywords: ume; plasma lipids; fecal output; intestinal flora; dietary fiber 


\section{Introduction}

Asian apricots have been used as daily foods and folk medicine in many Asian countries including Japan. The traditional drug Bainiku-ekisu, made from extracts of Japanese apricot (Prunus mume Sieb. et Zucc.), ume in Japanese, has been suggested to exert a powerful cardiovascular protective effect useful in the treatment of these diseases. It has also been reported that this drug markedly improves the fluidity of human blood. Bainiku-ekisu contains a novel compound, 1-[5-(2-formylfuryl)methyl] dihydrogen 2-hydroxypropane-1,2,3-tricarboxylate (mumefural), and a related compound, 5-hydroxymethyl- 2-furfural (HMF) [1]. Mumefural was found to markedly improve blood fluidity in all human subjects tested [1]. Mumefural is one of a number of compounds produced during thermal processing of ume extracts; thus the processing itself seems to be important for developing the functionality of this fruit.

Dietary fiber is known to affect the gut environment [2]. Addition of fiber to the diet is recommended for treating constipation. However, it has been reported that there are differences in the effects of various dietary fibers on gastrointestinal transit time in humans. Pectin (soluble dietary fiber) was found not to significantly alter the mean stool $\mathrm{pH}$, transit time or $24 \mathrm{~h}$ wet weight, while cellulose (insoluble dietary fiber) lowered the mean stool $\mathrm{pH}$ from 6.38 to 6.12 , decreased mean stool transit time by $27 \%$ and increased mean stool wet weight by $57 \%$ [3]. While dietary pectin may not significantly affect the gastrointestinal transit time, it is nevertheless known to affect the gut environment. Animals given soluble polysaccharides had plasma enteroglucagon levels significantly higher than animals given insoluble cellulose [4]. A 10\% pectin diet elicited a marked enlargement of the cecum, a drop in cecal $\mathrm{pH}$ and an increase in the volatile fatty acids (VFA) pool in rats [5]. Also in rats, a pectin-supplemented enteral diet was found to reduce the severity of methotrexate-induced enterocolitis [6].

Ume fruit contain both soluble and insoluble fiber. Recently, we developed a procedure to extract the dietary fiber from P. mume. While many functional characteristics have been reported for extracts of ume, as described above, there are few reports on the effects of the fiber extracts alone. In the present paper we have tested the hypothesis that fiber from this fruit changes gut function and intestinal flora in mice.

\section{Results and Discussion}

\subsection{General Observations}

No significant differences were observed between the UF and CF groups in final body weight (g) (UF 40.4 \pm 0.8 ; CF 40.9 \pm 0.4 ), in food consumption (g/day) (UF $4.4 \pm 0.16$; CF $4.4 \pm 0.17$ ), in visceral fat (g) (UF 2.74 \pm 0.24 ; CF $2.90 \pm 0.21$ ) or in liver weight (g) (UF $1.66 \pm 0.11$; CF $1.63 \pm 0.14$ ).

\subsection{Amount of Feces and Fecal Lipid Contents}

The UF diet significantly affected both the amount of feces and fecal lipid contents. The amount of feces (Figure 1) was significantly greater in the UF group than in the CF group. The fecal lipids content $(\% \mathrm{DW})$ of the feces sampled on the final day of the experiment were significantly greater in the UF group than in the CF group $(\mathrm{p}<0.01)$ (Figure 2). 
Figure 1. The amount of freeze-dried feces were significantly greater in the ume fiber (UF) group than in the cellulose $(\mathrm{CF})$ group. Values are means $\pm \mathrm{SE}(\mathrm{n}=7)$. The data were analyzed using $t$-test analysis. ${ }^{* *}$ Significantly different from the CF group $(\mathrm{p}<0.01)$; * Significantly different from the CF group $(\mathrm{p}<0.05)$.

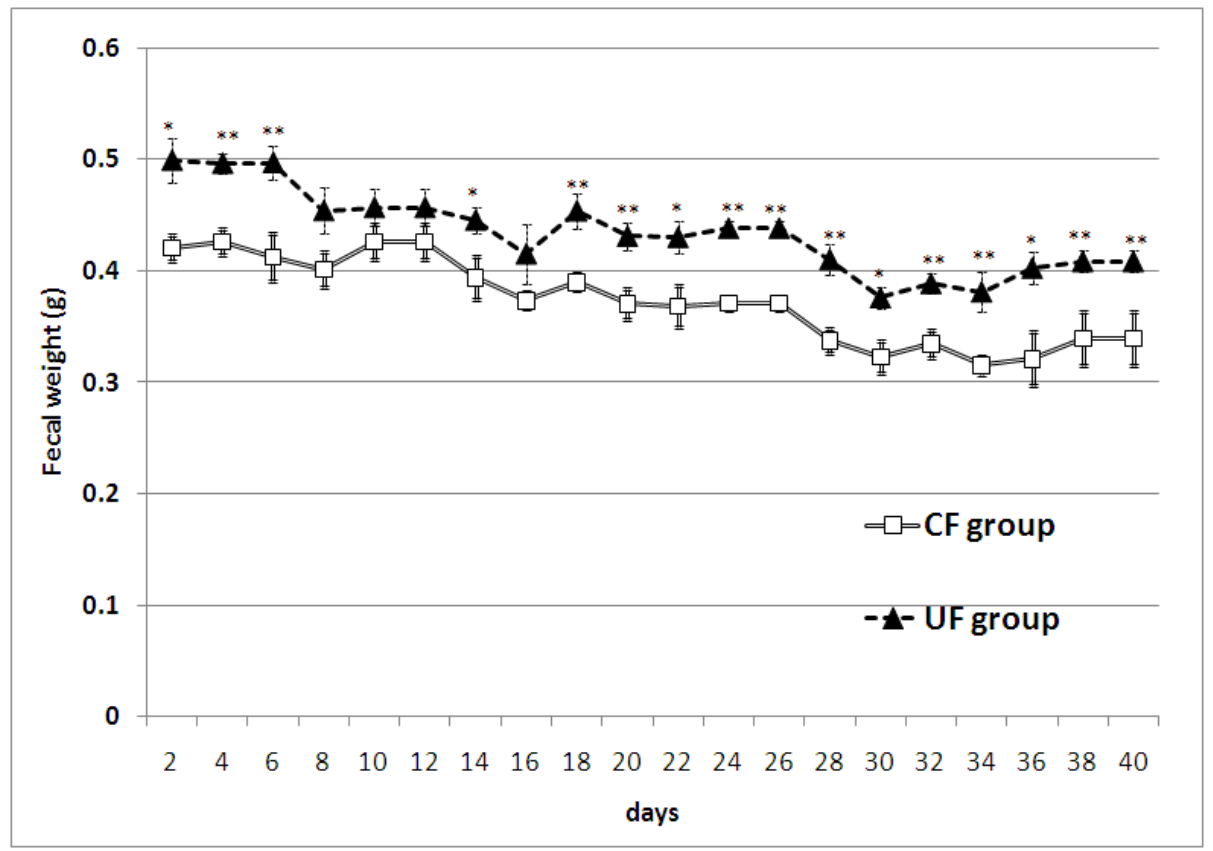

Figure 2. Fecal lipids contents (\%) from the feces sampled on the final day of the experiment. The fecal lipids content (\% DW) of the feces sampled on the final day of the experiment were significantly greater in the ume fiber (UF) group than in the cellulose (CF) group $(\mathrm{p}<0.01)$ Values are means \pm SE $(n=7)$. The data were analyzed using $t$-test analysis.

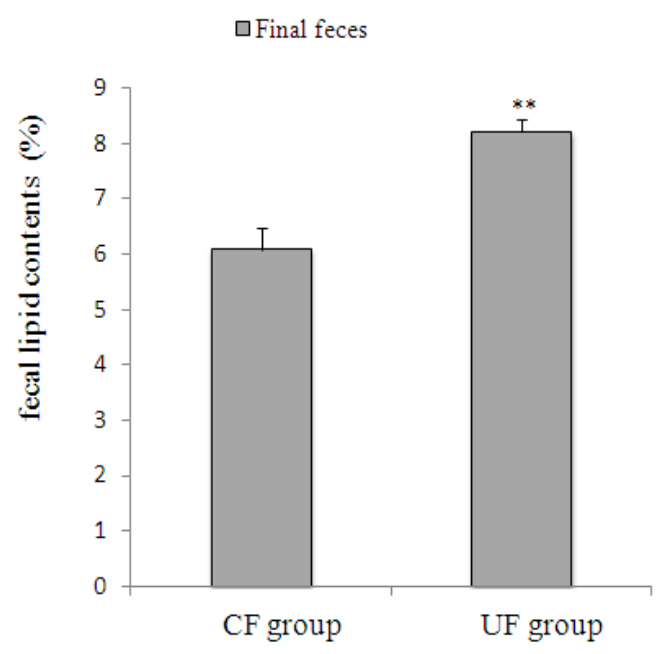

In the present study of the effects of fiber from the ume fruit on mice gut function and microflora, dry fecal weight was significantly increased in the UF compared to the CF group. It has been reported that diets containing wheat bran increased wet (by 67\%) and dry (by 74\%) fecal weight. However, diets containing soluble fiber in the form of pectin did not influence dry weight [7]. In our experiment, the CF diet did not contain soluble dietary fiber, while ume fiber contains both insoluble and soluble 
fibers. Insoluble dietary fiber plus soluble dietary fiber might effectively increase the amount of fecal output in the UF group. In our experiment, both UF and CF diets contained high fat (10\%). Generally, incidence of tumors increases with increasing fat content in the diet [8]. It has been reported that tumorigenesis in rats was enhanced by increased fat content of the diet [9] while wheat bran was found to reduce tumor development in these animals [10]. In the latter report, the authors suggested that the effect of wheat bran could be due to a reduction of exposure of the colonic epithelium to carcinogens and promoters that are presumed to be necessary for tumor development. It has been reported that the dietary fiber inulin modulates parameters of colon cancer risks in human and animals colon cells. The mechanisms responsible possibly include reduction of exposure to risk factors and suppression of tumor cell survival. The dilution of carcinogenic factors to lower their contact with colon cells is considered to be one of the most important dietary strategies [11]. It has also been reported that increased incidence rate of colorectal tumors due to the intake of a soluble dietary fiber in chemically-induced rat carcinogenesis can be suppressed by partially substituting an insoluble fiber [12]. Due to its fecal bulking effect, and its mixture of insoluble and soluble polymers, ume fiber may thus be an interesting preventive dietary supplement against cancer of the colon. The soluble and insoluble dietary fiber seem to differently affect body weight in a long-term feeding. It has been reported that soluble $v$ s. insoluble dietary fiber added to a high-fat, Western-style diet differently affected body weight [13]. In this report, a long-term study investigated potential protective effects of adding soluble guar fiber $(10 \% \mathrm{w} / \mathrm{w})$ vs. insoluble cereal fiber $(10 \% \mathrm{w} / \mathrm{w})$ to an isoenergetic and macronutrient matched high-fat diet in obesity-prone C57BL/6J mice. After 45 weeks, mice fed soluble vs. insoluble fiber showed significantly increased body weight $(41.8+/-3.0 v$ s. $33.6+/-1.5 \mathrm{~g}, \mathrm{p}=0.03)$. In our study, there were no significant differences in final body weight between the UF and CF groups. It is expected that at least the soluble fraction of ume fiber shows stronger fermentation in comparison to cellulose. However, the experimental period was relatively short-term (40 days). So, further studies are needed to clarify the effects of ume fiber on body weight and obesity in long-term studies.

\subsection{Plasma Total Cholesterol, Triglyceride, Phospholipids, NEFA and Plasma Glucose}

At the end of the diet feeding period, the mice were anesthetized with diethylether and blood samples were taken from the abdominal aorta and placed in heparinized tubes. The plasma was separated from whole blood by centrifugation and used for analysis of plasma triglyceride, total cholesterol, phospholipids, NEFA and glucose. No significant differences in the plasma cholesterol (UF $205.2 \pm 27.0 \mathrm{mg} / \mathrm{dL}$; CF $263.3 \pm 46.7 \mathrm{mg} / \mathrm{dL}$ ), plasma triglyceride (UF $160.4 \pm 10.7 \mathrm{mg} / \mathrm{dL}$; CF $158.7 \pm 23.1 \mathrm{mg} / \mathrm{dL}$ ) or plasma phospholipids concentrations (UF $255.9 \pm 21.8 \mathrm{mg} / \mathrm{dL}$; CF $276.1 \pm 24.0 \mathrm{mg} / \mathrm{dL}$ ) were observed between the two groups. However, plasma NEFA concentrations tended to be lower in the UF than in the CF group (Figure 3) $(\mathrm{p}=0.058)$. No significant differences between the two groups were observed for plasma glucose (UF $224.1 \pm 24.2 \mathrm{mg} / \mathrm{dL}$; CF $243.3 \pm 30.7 \mathrm{mg} / \mathrm{dL}$ ). In the present study, the increased fecal lipid content of the UF group compared to controls is likely to be related to the group's lower plasma NEFA concentration. Pearson product-moment correlation coefficient between the plasma NEFA and fecal lipid content of two dietary groups were analyzed. Negative correlation $(\mathrm{r}=-0.632)$ was observed between the plasma NEFA and fecal lipid content of the two dietary groups. 
Figure 3. Plasma non-esterified fatty acids (NEFA) concentrations of mice in the ume fiber (UF) and cellulose (CF) groups. Values are means \pm SE $(n=7)$. The data were analyzed using $t$-test analysis $(\mathrm{p}=0.058)$.

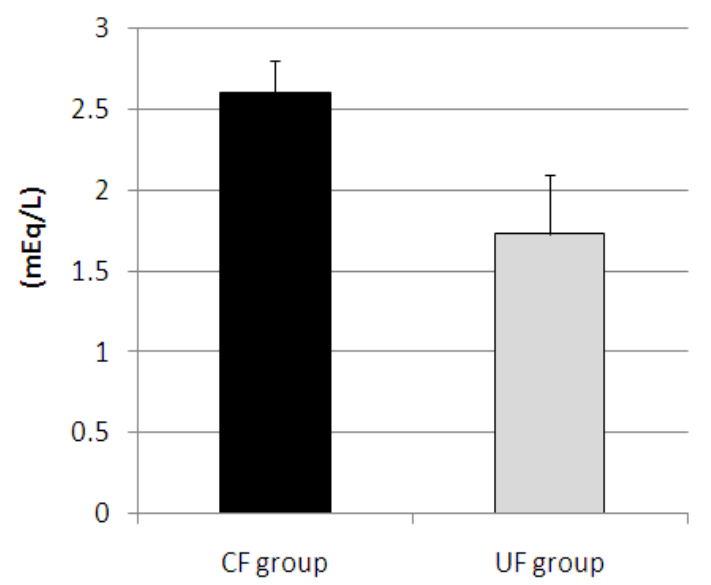

\subsection{Effects of Diet on Cecal Flora of Mice}

The compositions of the phylogenetic groups of cecal flora differed between the two dietary groups (Figure 4). The occupation ratios of Bacteroides and Clostridium cluster IV were significantly greater in the UF than in the CF group ( $p<0.01$ ). It has been confirmed that intestinal microbiota predominantly consist of the members of approximately ten phylogenetic bacterial groups and that these bacterial groups can be distinguished by the T-RFLP system developed by Nagashima et al. [14,15]. There were significant differences in the composition of the intestinal flora between the two groups. It has been shown that consumption of apple pectin ( $7 \%$ in the diet) increases the population of butyrate- and beta-glucuronidase producing Clostridiales, and decreases the population of specific species within the Bacteroidetes group in the rat gut [16]. However, in our results, occupation ratios of Bacteroides and Clostridium cluster IV were significantly greater in the UF group. Different types of fiber may differently affect the intestinal flora of mice. Recently, much attention has been focused on the relation between intestinal flora and obesity. It has been reported that the amounts of Bacteroides are negatively correlated with fat pad mass, body mass and body-mass gain in rats [17]. Studies on human volunteers have revealed that obesity is associated with changes in the relative abundance of the two dominant bacterial divisions, the Bacteroidetes and the Firmicutes [18]. In rats, the Bacteroides group has been reported to affect the body mass and fat pad mass. In our experiment, despite the observed difference in bacterial populations, no such physical differences were observed between the two dietary groups. However, it is possible that the differences in bacterial composition observed were due to the differences of the fecal lipids content between two dietary groups. For our future research in this field, an investigation of the relationship between high fiber diets, including ume fiber, fat pad mass and the obesity phenotype and intestinal microflora, would be a most useful contribution. 
Figure 4. Composition of cecal intestinal microflora of mice in the ume fiber (UF) and cellulose (CF) groups. OTUs (operational taxonomic units), which correspond to either T-RFs (terminal restriction fragments) or T-RF clusters, detected by T-RFLP analysis. Values are means $\pm \operatorname{SE}(\mathrm{n}=7) .{ }^{*}$ Significantly different $(\mathrm{p}<0.01)$ from the CF group. The data were analyzed using $t$-test analysis. The letters correspond to the following phylogenetic bacterial groups: (A) Bacteroides, Clostridium cluster IV (OTUs 370); (B) Clostridium cluster IV (OTUs 168, 749); (C) Clostridium cluster IX, Megamonas (OTUs 110); (D) Clostridium cluster XI (OTUs 338); (E) Clostridium subcluster XIVa (OTUs 106, 494, 505, 517, 754, 955, 990); (F) Clostridium cluster XI, Clostridium subcluster XIVa (OTUs 919); (G) Clostridium subcluster XIVa, Enterobacteriales (OTUs 940).; (H) Clostridium cluster XVIII (OTUs 423, 650); (I) Bacteroides (OTUs 469, 853); (J) Bifidobacterium (OTUs 124); (K) Lactobacillales (OTUs 332, 520, 657); (L) Prevotella (OTUs 137, 317); (M) Others.

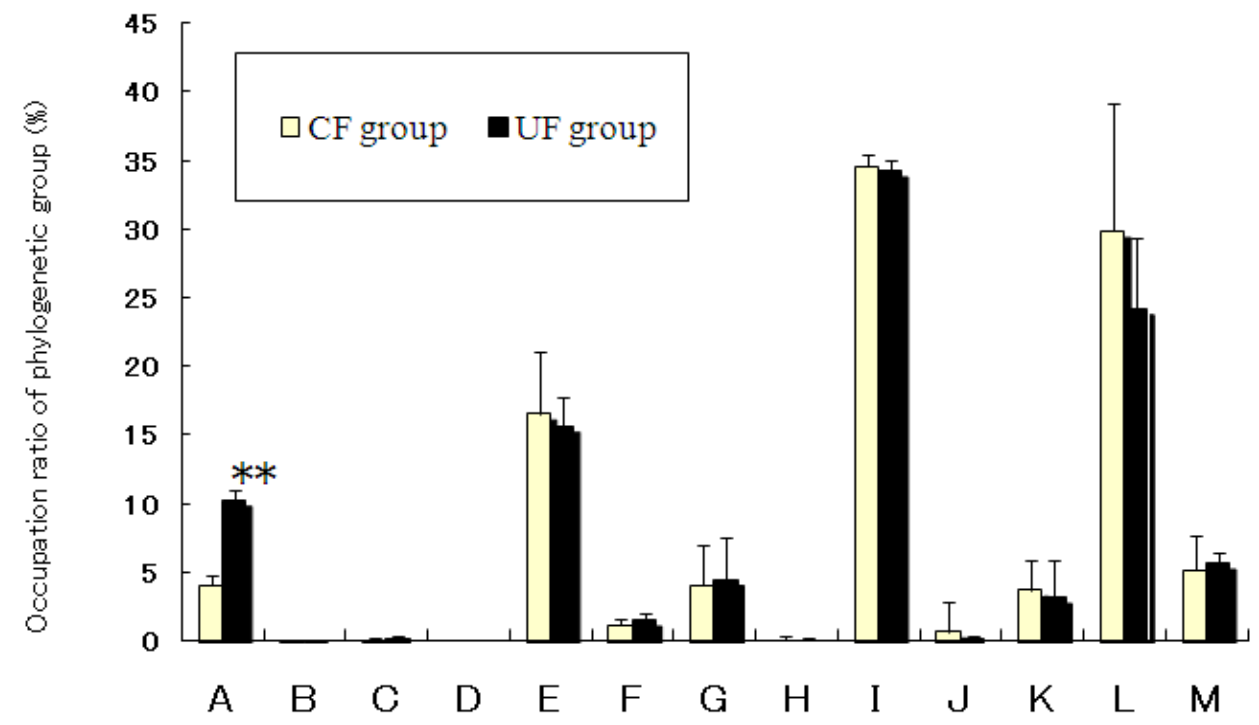

\section{Experimental Section}

\subsection{Production of Fiber from Fruit of Prunus mume}

The process of manufacturing ume fiber from Prunus mume is summarized in Figure 5.

Fiber from ume was prepared as follows. Whole ume (100 g), from which seeds were removed, were mashed to a puree. The puree $(87.8 \mathrm{~g}$ ) was then centrifuged at $8,000 \mathrm{rpm}$ for $20 \mathrm{~min}$ (himac CR22G, HITACHI Co., Ltd., Tokyo, Japan). The sedimented material $(23.5 \mathrm{~g})$ was dried at $60{ }^{\circ} \mathrm{C}$ with a food drier (far-infrared radiation food drier vivi-9, Vianove Co., Ltd.) until the moisture content was less than 5\%. The dried residue was ground down by mill (Wonder Crush/Mill WDL-1, Osaka Chemical Co., Ltd., Osaka, Japan) until the particle diameter of the powder was below 60 meshes. Finally, $3.8 \mathrm{~g}$ of ume fiber was obtained as a powder. 
Figure 5. The method of manufacturing ume fiber.

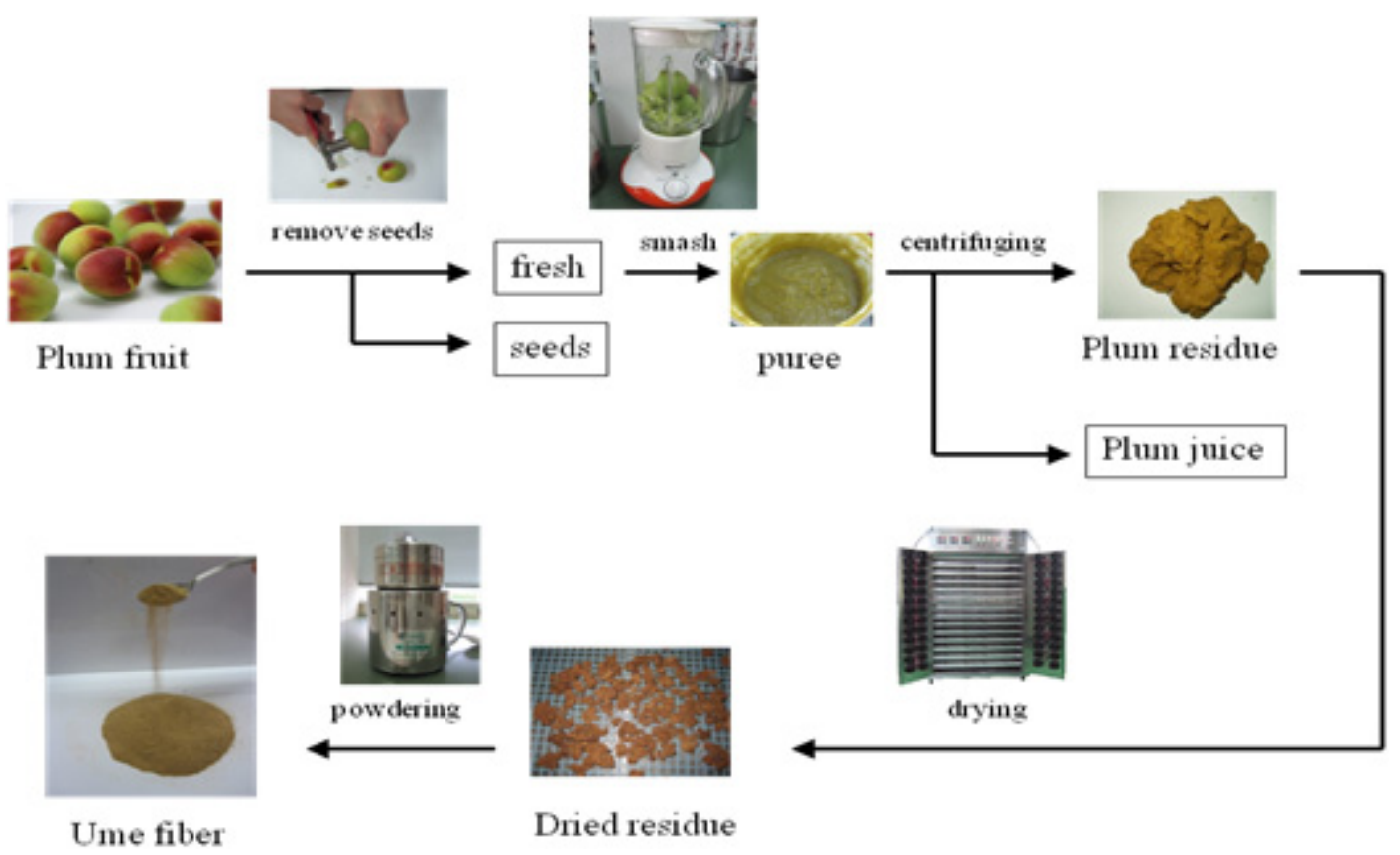

\subsection{Treatment of Animals}

Fourteen male Crj: CD-1 (ICR) mice (seven weeks old) were purchased from Charles River Japan, Inc. (Kanagawa, Japan). All mice were specific pathogen-free (SPF), and the animals were housed under conventional conditions in our laboratory. The mice were randomly divided into two groups of seven animals each and housed in suspended stainless-steel cages with wire mesh bottoms, in a room kept at $24 \pm 0.5{ }^{\circ} \mathrm{C}$, relative humidity $65 \%$, with $12 \mathrm{~h}$ periods of light and dark. The mice were fed an AIN-93M diet for one week. The diet was then replaced with an ume fiber (UF) or cellulose (CF) diet (control) for 40 days. All mice were pair-fed. Table 1 presents the composition of each diet. The cellulose powder used in the CF diet was purchased from Oriental Yeast Co., Ltd.

Table 1. Composition of the experimental diet.

\begin{tabular}{lccc}
\hline Ingredient (g/kg diet) & AIN-93M & UF diet & CF diet \\
\hline Corn starch & 465.692 & 388.246 & 405.686 \\
Casein & 140 & 129.52 & 140 \\
$\alpha$-Corn starch & 155 & 155 & 155 \\
Sucrose & 100 & 100 & 100 \\
Rice bran oil & - & 97.52 & 100 \\
Soy bean oil & 40 & - & - \\
Cellulose & 50 & - & 50 \\
Ume fiber & - & 80.4 & - \\
Mineral mix (AIN-93M-Mix) & 35 & 35 & 35 \\
Vitamin mix (AIN-93-Mix) & 10 & 10 & 10 \\
L-Cystine & 1.8 & 1.8 & 1.8 \\
Choline Bitartrate & 2.5 & 2.5 & 2.5 \\
Tert-butylhydroquinone & 0.008 & 0.014 & 0.014 \\
\hline
\end{tabular}


The analysis of ume fiber was conducted by Japan Food Research Laboratories according to the manual of analytical methods for standard tables of food composition in Japan (Fifth Revised and Enlarged Edition). The nutritional components of ume fiber were as follows: moisture, 4.2\%; protein, $13.1 \%$; lipids, $3.1 \%$; ash, $2.2 \%$; carbohydrate, $15.2 \%$, dietary fiber, $62.2 \%$ (soluble dietary fiber, $3.1 \%$; insoluble dietary fiber, $59.1 \%$ ). According to this information, we adjusted the composition of the UF or CF diets so that each had similar contents of fiber, protein, lipids and carbohydrate. Body weight, food consumption and amount of feces were measured during the experiment. Feces were dried by freeze dryer FD-1000 (Tokyo Rikakikai Co., Ltd., Tokyo, Japan) for 24 hr. Trap cooling temperature was $-45^{\circ} \mathrm{C}$. Amounts of freeze-dried feces were also measured during the experiment.

\subsection{Analysis of ume Fiber, Mice Feeding Conditions and Sampling}

At the end of the diet feeding period, the mice were anesthetized with diethylether and blood samples were taken from the abdominal aorta and placed in heparinized tubes. The plasma was separated from whole blood by centrifugation and stored at $-80{ }^{\circ} \mathrm{C}$ for later analysis of plasma triglyceride, total cholesterol, phospholipids, NEFA and glucose. The mice were then euthanized with diethylether. The liver and cecal contents were collected. Cecal contents were stored at $-80{ }^{\circ} \mathrm{C}$ for analysis of intestinal microflora by T-RFLP. The liver samples and visceral fat were weighed. All procedures involving mice in this study were approved by the Animal Care Committee of the National Food Research Institute in Japan, in accordance with the "Guidelines for Animal Care and Experimentation" of the National Food Research Institute in Japan. The animal studies were reviewed and approved by the Animal Care and Use Committee of the National Food Research Institute, and the National Agriculture and Food Research Organization (NARO), Japan.

\subsection{Measurement of Plasma Cholesterol, Triglyceride, Phospholipids, NEFA and Plasma Glucose}

The following tests were performed with Wako kits obtained from Wako Pure Chemical Industries Ltd., Osaka, Japan. Total plasma cholesterol concentrations were measured using a cholesterol E-test Wako kit based on cholesterol oxidase [19]. Plasma triglyceride concentrations were measured using a triglyceride E-test Wako kit based on the glycerol-3-phosphate oxidase method [20]. Plasma phospholipid concentrations were measured using a phospholipid C-test Wako kit based on the choline oxidase method [21]. Plasma NEFA were measured using a NEFA C-test Wako kit based on the acyl-CoA synthase (ACS) and acyl-CoA oxidase (ACO) method. The plasma glucose concentrations were measured using a glucose $\mathrm{C} 2$-test Wako kit based on the mutarotase glucose oxidase method.

\subsection{Fecal Lipid Extraction}

Feces were dried by freeze dryer FD-1000 (Tokyo Rikakikai Co., Ltd., Tokyo, Japan) for 24 h. Trap cooling temperature was $-45^{\circ} \mathrm{C}$. After drying, feces were milled by food mill TML17 (TESCOM Co., Ltd., Tokyo, Japan) for $30 \mathrm{~s}$. Fecal lipid was extracted from the fecal powder by the Bligh and Dyer method [22]. 


\subsection{DNA Extraction from Cecal Contents}

DNA extractions from cecal contents were conducted according to the Matsuki's method [23]. Cecal samples $(20 \mathrm{mg})$ were washed three times by suspending them in $1.0 \mathrm{~mL}$ of phosphate-buffered saline and centrifuging each preparation at $14,000 \mathrm{~g}$ in order to remove possible PCR inhibitors. Following the third centrifugation the cecal pellets were resuspended in a solution containing $0.2 \mathrm{~mL}$ of phosphate-buffered saline and $250 \mu \mathrm{L}$ extraction buffer (200 mM Tris-HCl, $80 \mathrm{mM}$ EDTA; pH 9.0) and $50 \mu \mathrm{L} 10 \%$ sodium dodecyl sulfate. Three hundred milligrams of glass beads (diameter $0.1 \mathrm{~mm}$ ) and $500 \mu \mathrm{L}$ of buffer-saturated phenol were added to the suspension, and the mixture was vortexed vigorously for $60 \mathrm{~s}$ using a Mini Bead-Beater (BioSpec Products Inc., Bartlesville, OK) at a power level of $4800 \mathrm{rpm}$. Following centrifugation at $14,000 \mathrm{~g}$ for $5 \mathrm{~min}, 400 \mu \mathrm{L}$ of the supernatant was collected. Phenol-chloroform-isoamyl alcohol extractions were then performed, and $250 \mu \mathrm{L}$ of the supernatant was subjected to isopropanol precipitation. Finally, the DNA was suspended in $1 \mathrm{~mL}$ Tris-EDTA buffer. The DNA preparation was adjusted to a final concentration of $10 \mu \mathrm{g} / \mathrm{mL}$ in TE and checked by $1.5 \%$ agarose gel electrophoresis.

\subsection{PCR Conditions and Restriction Enzyme Digestion}

The PCR mixture $(25 \mu \mathrm{L})$ was composed of EX Taq buffer, $2 \mathrm{mM} \mathrm{Mg}^{2+}$ and each deoxynucleoside triphosphate at a concentration of $200 \mu \mathrm{M}$. The amount of cecal DNA was $10 \mathrm{ng}$. The primers used were 5' HEX-labeled 516f (5'-TGCCAGCAGCCGCGGTA-3') and 1510r (5'-GGTTACCTTGTTACGACTT-3') at a concentration of $0.10 \mu \mathrm{M}$, template DNA and $0.625 \mathrm{U}$ of TaKaRa EX Taq DNA polymerase (Takara Bio Inc., Otsu, Japan). This process was carried out using the Dice PCR System (Takara Bio Inc.). Amplification was performed with one cycle at $95{ }^{\circ} \mathrm{C}$ for $15 \mathrm{~min}$, followed by 30 cycles at $95{ }^{\circ} \mathrm{C}$ for $30 \mathrm{~s}, 50{ }^{\circ} \mathrm{C}$ for $30 \mathrm{~s}, 72{ }^{\circ} \mathrm{C}$ for $1 \mathrm{~min}$, and finally one cycle at $72{ }^{\circ} \mathrm{C}$ for $10 \mathrm{~min}$. The amplification products were subjected to gel electrophoresis in $1.5 \%$ agarose followed by ethidium bromide staining. The PCR products were purified using QIAquick spin columns (Qiagen KK, Tokyo, Japan) according to the manufacturer's instructions. The purified DNA was treated with $2 \mathrm{U}$ of Bs $1 I$ (New England Biolabs) for $3 \mathrm{~h}$, at $55^{\circ} \mathrm{C}$ [14].

\subsection{T-RFLP Analysis}

The fluorescently labeled T-RFs were analyzed by electrophoresis on an ABI PRISM 310 Genetic Analyzer automated sequence analyzer (Applied Biosystems) in GeneScan mode. The restriction enzyme digestion mixture $(2 \mu \mathrm{L})$ was mixed with $0.5 \mu \mathrm{L}$ of MapMarker 1000 size standard (BioVentures, Inc.) and $12 \mu \mathrm{L}$ of deionized formamide. The mixture was denatured at $96{ }^{\circ} \mathrm{C}$ for $2 \mathrm{~min}$ and immediately chilled on ice. The injection time was $30 \mathrm{~s}$ for analysis of T-RFs from the digestion with Bsl $I$. The run time was $40 \mathrm{~min}$. The lengths and peak areas of T-RFs were determined with the GeneMapper software. From the predominant operational taxonomic units (OTUs, which correspond to either T-RFs or T-RF clusters) that were detected in the T-RFLP profiles, phylogenetic groups of intestinal flora were identified [14,15]. 


\subsection{Statistics}

The data are expressed as the mean \pm standard error (SE). All data were analyzed using the Sigma Plot 11 (Systat Software, Inc., CA, USA). Pearson product-moment correlation coefficient between the plasma NEFA and fecal lipid content of two dietary groups were analyzed. The remaining data were analyzed using $t$-test analysis. Statistical significance was reached with a $\mathrm{P}$ value of less than 0.05 .

\section{Conclusions}

In conclusion, fecal output was significantly increased in the UF group compared to the CF group. Both fecal weight and fecal lipid concentration on the final day were significantly greater in the UF than in the CF groups. There were significant differences in the composition of the intestinal flora between two groups. Occupation ratios of Bacteroides and Clostridium cluster IV were significantly greater in the UF group. The characteristics described for ume fiber, including its feces bulking effects, suggest that this new fiber possesses the fecal lipid excretion effects and feces bulking effects.

\section{Acknowledgements}

This study was financially supported by the Ministry of Economy, Trade and Industry of Japan.

\section{References}

1. Chuda, Y.; Ono, H.; Ohnishi-Kameyama, M.; Matsumoto, K.; Nagata, T.; Kikuchi, Y. Mumefural, citric acid derivative improving blood fluidity from fruit-juice concentrate of Japanese apricot (Prunus mume Sieb. et Zucc). J. Agr. Food. Chem. 1999, 47, 828-831.

2. Weickert, M.O.; Pfeiffer, A.F. Metabolic effects of dietary fiber consumption and prevention of diabetes. J. Nutr. 2008, 138, 439-442.

3. Hillman, L.; Peters, S.; Fisher, A.; Pomare, E.W. Differing effects of pectin, cellulose and lignin on stool pH, transit time and weight. Br. J. Nutr. 1983, 50, 189-195.

4. Johnson, I.T.; Gee, J.M.; Brown, J.C. Plasma enteroglucagon and small bowel cytokinetics in rats fed soluble nonstarch polysaccharides. Am. J. Clin. Nutr. 1988, 47, 1004-1009.

5. Demigné, C.; Levrat, M.A.; Rémésy, C. Effects of feeding fermentable carbohydrates on the cecal concentrations of minerals and their fluxes between the cecum and blood plasma in the rat. J. Nutr. 1989, 119, 1625-1630.

6. Mao, Y.; Kasravi, B.; Nobaek, S.; Wang, L.Q.; Adawi, D.; Roos, G.; Stenram, U.; Molin, G.; Bengmark, S.; Jeppsson, B. Pectin-supplemented enteral diet reduces the severity of methotrexate induced enterocolitis in rats. Scand. J. Gastroenterol. 1996, 31, 558-567.

7. Armstrong, E.F.; Eastwood, M.A.; Brydon, W.G. The influence of wheat bran and pectin on the distribution of water in rat caecal contents and faeces. Br. J. Nutr. 1993, 69, 913-920.

8. Wijnands, M.V.; Appel, M.J.; Hollanders, V.M.; Woutersen, R.A. A comparison of the effects of dietary cellulose and fermentable galacto-oligosaccharide, in a rat model of colorectal carcinogenesis: Fermentable fibre confers greater protection than non-fermentable fibre in both high and low fat backgrounds. Carcinogenesis 1999, 20, 651-656. 
9. Rao, C.V.; Hirose, Y.; Indranie, C.; Reddy, B.S. Modulation of experimental colon tumorigenesis by types and amounts of dietary fatty acids. Cancer Res. 2001, 61, 1927-1933.

10. Zoran, D.L.; Turner, N.D.; Taddeo, S.S.; Chapkin, R.S.; Lupton, J.R. Wheat bran diet reduces tumor incidence in a rat model of colon cancer independent of effects on distal luminal butyrate concentrations. J. Nutr. 1997, 127, 2217-2225.

11. Pool-Zobel, B.L.; Sauer, J. Overview of experimental data on reduction of colorectal cancer risk by inulin-type fructans. $J$. Nutr. 2007, 137, 2580S-2584S.

12. Okazaki, H.; Nishimune, T.; Matsuzaki, H.; Miura, T.; Morita, S.; Yanagimoto, Y.; Yamagishi, H.; Yamada, K.; Ikegami, S. Increased incidence rate of colorectal tumors due to the intake of a soluble dietary fiber in rat chemical carcinogenesis can be suppressed by substituting partially an insoluble dietary fiber for the soluble one. Int. J. Cancer 2002, 100, 388-394.

13. Isken, F.; Klaus, S.; Osterhoff, M.; Pfeiffer, A.F.; Weickert, M.O. Effects of long-term soluble vs. insoluble dietary fiber intake on high-fat diet-induced obesity in C57BL/6J mice. J. Nutr. Biochem. 2010, 21, 278-284.

14. Nagashima, K.; Hisada, T.; Sato, M.; Mochizuki, J. Application of new primer-enzyme combinations to terminal restriction fragment length polymorphism profiling of bacterial populations in human feces. Appl. Environ. Microbiol. 2003, 69, 1251-1262.

15. Nagashima, K.; Mochizuki, J.; Hisada, T.; Suzuki, S.; Shimomura, K. Phylogenetic analysis of $16 \mathrm{~S}$ ribosomal RNA gene sequences from human fecal microbiota and improved utility of terminal restriction fragment length polymorphism profiling. Biosci. Microflora. 2006, 25, 99-107.

16. Licht, T.R.; Hansen, M.; Bergström, A.; Poulsen, M.; Krath, B.N.; Markowski, J.; Dragsted, L.O.; Wilcks, A. Effects of apples and specific apple components on the cecal environment of conventional rats: Role of apple pectin. BMC Microbiol. 2010, 10, 13.

17. Mozes, S.; Bujnáková, D.; Sefcíková, Z.; Kmet V. Intestinal microflora and obesity in rats. Folia. Microbiol. (Praha.) 2008, 53, 225-228.

18. Turnbaugh, P.J.; Ley, R.E.; Mahowald, M.A.; Magrini, V.; Mardis, E.R.; Gordon, J.I. An obesity-associated gut microbiome with increased capacity for energy harvest. Nature 2006, 444, 1027-1031.

19. Allain, C.C.; Poon, L.S.; Chan, C.S.; Richmond, W.; Fu, P.C. Enzymatic determination of total serum cholesterol. Clin. Chem. 1974, 20, 470-475.

20. Spayd, R.W.; Bruschi, B.; Burdick, B.A.; Dappen, G.M.; Eikenberry, J.N.; Esders, T.W.; Figueras, J.; Goodhue, C.T.; LaRossa, D.D.; Nelson, R.W.; Rand, R.N.; Wu, T.W. Multilayer film elements for clinical analysis: Applications to representative chemical determinations. Clin. Chem. 1978, 24, 1343-1350.

21. Takayama, M.; Itoh, S.; Nagasaki, T.; Tanimizu, I. A new enzymatic method for determination of serum choline-containing phospholipids. Clin. Chim. Acta 1977, 79, 93-98.

22. Bligh, E.G.; Dyer, W.J. A rapid method of total lipid extraction and purification. Can. J. Biochem. Physiol. 1959, 37, 911-917. 
23. Matsuki, T. Procedure of DNA extraction from fecal sample for the analysis of intestinal microflora. J. Intestinal. Microbiol. 2006, 20, 259-262.

(C) 2011 by the authors; licensee MDPI, Basel, Switzerland. This article is an open access article distributed under the terms and conditions of the Creative Commons Attribution license (http://creativecommons.org/licenses/by/3.0/). 